\title{
IMPLEMENTATION OF MULTIOBJECTIVE OPTIMIZATION PROCEDURES AT THE PRODUCT DESIGN PLANNING STAGE
}

\author{
M. Yoshimura ${ }^{1}$ \\ ${ }^{1}$ Department of Aeronautics and Astronautics, Mechanical Engineering Division, Graduate \\ School of Engineering, Kyoto University, yoshimura@prec.kyoto-u.ac.jp
}

\begin{abstract}
In order to obtain maximally innovative and successful product designs, the utilization of optimization strategies at the product design planning stage is of prime importance, and the methods proposed in this paper enable multiobjective optimization technologies to be effectively applied. The necessity and effectiveness of utilizing optimization techniques at the product design planning stage are first explained, and the features that this requires are then clarified. Optimization solutions provided at the product design planning stage, while far from final, can nevertheless be used to obtain guidelines for more preferable product designs. For this purpose, even if characteristics evaluated at the product design planning stages are simplified and/or idealized, the interrelationships among all related characteristics should be simultaneously and thoroughly explored. The successful application of optimization techniques at the product design planning stage requires the rapid presentation and evaluation of a variety of alternative designs, a deeper understanding of the reasons for the optimized designs that are developed, and breakthrough of the initial optimized design solutions, so that the most effective design can ultimately be implemented in a manufactured product. This paper proposes multiobjective design optimization methodologies and procedures, utilized at the product design planning stage, to achieve these goals.
\end{abstract}

keywords: Product design planning stage, Multiobjective optimization, Pareto optimum solutions, Comparison of alternative designs, Hierarchical optimization problem, Rapid evaluation, Deeper insight into design solutions

\section{Introduction}

Today's rigorous manufacturing environments require the application of optimization techniques from wider points of view. To accomplish this, strategic utilization of optimization techniques at the product design planning stage, a process far upstream of product manufacturing, is essential. In this paper, the significance of conducting optimization at the product design planning stage

Please use the following format when citing this chapter:

Author(s) [insert Last name, First-name initial(s)], 2006, in IFIP International Federation for Information Processing, Volume 199, System Modeling and Optimization, eds. Ceragioli F., Dontchev A., Furuta H., Marti K., Pandolfi L., (Boston: Springer), pp. [insert page numbers]. 
is first clarified. Next, problem areas concerning the use of optimization techniques at the product design planning stage, and desirable features that such techniques should offer, are described.

At the product planning stage, principal product performances for the product should be considered and evaluated, and the conflicting relationships among the characteristics should be quickly but roughly evaluated. Many alternative designs are usually generated and compared at this stage and, for the most part, multiobjective optimization methods are applied. In multiobjective optimization problems, a Pareto optimum solution set, namely a set of feasible solutions for each of which there exists no other feasible solution that yields an improvement in one objective without causing degradation in at least one other objective, is obtained to evaluate conflicting objectives of the design optimization problem at hand [1] [2]. In order to effectively apply multiobjective optimization methods to the product planning stage, new methods need to be developed, to incorporate fundamental improvements in the multiobjective optimization. This paper presents methodologies for executing optimization at the product design planning stage, and several applied examples are given. Several methods developed by the author and his colleagues are organized and presented so that they can be effectively applied at the product design planning stage.

\section{Significance of Optimization at the Product Design Planning Stage}

\subsection{Features of the product design planning stage in manufacturing processes}

Figure 1 shows the sequence of manufacturing processes, where the product design planning is the first step. The product design planning, located furthest upstream, determines practically all of the downstream manufacturing process details. Current design environments require careful consideration not only of increasingly demanding requirements for product performances, qualities, and product cost, but also many other factors such as the product's environmental impact, lifecycle and recycling, and safety. Aggressive and relentless competition among companies developing new products under such circumstances makes the application of optimization strategies throughout product manufacturing processes a practical requirement. Particularly important to successful manufacturing is the application of optimization methods that start from the initial planning stages of product design. 


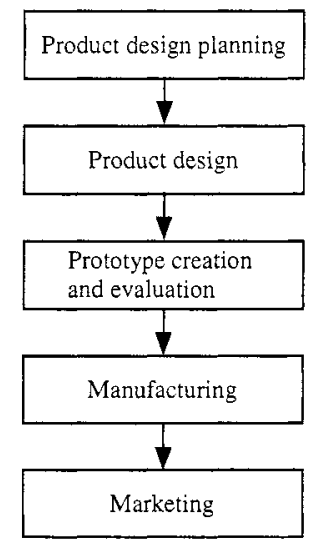

Figure 1. The sequence of manufacturing processes

\subsection{Features of design optimization applied at the product design planning stage}

The most effective application of optimization methods is based on careful inquiry and consideration of the features of the product design planning stage. This stage is more or less equivalent to the conceptual design, or fundamental design stage, where details of the product design have not yet been determined but various conceptual designs are considered, compared, and evaluated. This is when the design specifications for the product and its requirements and characteristics are usually given. Also, the principal characteristics used for evaluating the product performances can be defined. At the product design planning stage, the product performance and the product manufacturing cost for the entire product should be roughly evaluated even if the estimated values of the characteristics are imprecise.

At the product design planning stage, all characteristics should be systematically evaluated, and selection of the design candidates from among many design alternatives should be conducted, using an optimization procedure. Multiobjective optimization techniques can be effectively applied to systematical evaluations of the characteristics being regarded.

Solutions of optimization at the product design planning stages can be used for obtaining guidelines for product designs. For the purposes, the facts that characteristics considered at the product design planning stages may be simple and /or idealized can be acceptable. But, the relationships among the all related characteristics should be totally inquired.

When existing design solutions are available, searching methods that can achieve breakthrough or improved solutions are needed. 
Figure 2 shows the conflicting relationship between two performance characteristics, $f_{1}$ and $f_{2}$. A larger value is preferable for each of these performance characteristics. The shaded area corresponds to the feasible region formed by design solutions that can be realized using present technologies and knowledge. The line PQ corresponds to the Pareto optimum solution set of global optimum solutions, achieved by concurrent optimization of all related characteristics. Product designers generally look for practical design solutions on a Pareto optimum solution line. From the Pareto optimum solution set, the most suitable solution is selected by considering the design requirements and the product environments.

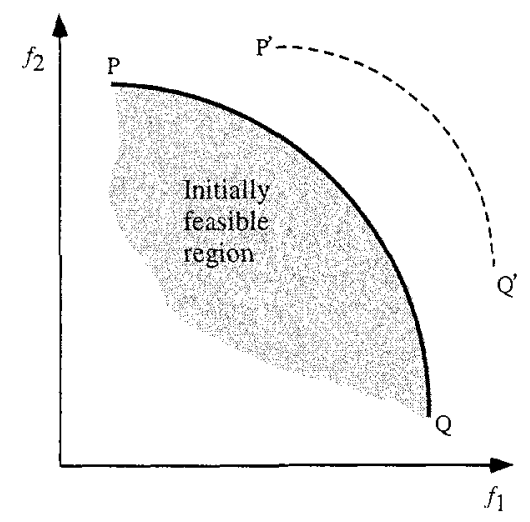

Figure 2. Concept of the proposed methods

If an alternative design results in a new optimum solution line, such as P'Q' shown in Fig.2, it can represent an improved new Pareto optimum solution set line. The display of such Pareto optimum solution sets is effective since designers can quickly understand the features of entire solutions.

\subsection{Requirements for applying optimization techniques at the Product Design Planning Stage}

In order to apply optimization techniques at the product design planning stage, the following points should be realized:

1 Since designers usually have a wide range of initial ideas, numerous alternative designs should be quickly formulated and effectively compared with each other, so that the most suitable small number of designs can be selected.

2 The many performance characteristics of the designs at hand must be concurrently evaluated and optimized. 
Multiobjective optimization methods that incorporate new and improved advanced techniques can be applied to achieve the foregoing points. The details of such methods are explained below, along with some applied examples that illustrate their application.

\section{Methodologies of Design Optimization at the Product Design Planning Stage}

\subsection{Comparison of many alternative designs}

In the first method, many design alternatives in the multiobjective functions space are displayed in detail, and a relational tree diagram of design alternatives is shown to aid a deeper understanding of the optimized solutions [3]. To explain the process, the design of industrial multi-link manipulators that are used to transport an object in a workspace within a given operational time is used.

Alternative designs are constructed from a group of modules. Basic module is one link mechanism with a motor corresponding to a minimum unit of linked mechanisms. To create systems of practical complexity, we add design variables and increase the number of degrees of freedom. Here, two kinds of operators, operators 1 and 2, that control modules during the process of constructing a more complex system are introduced. Operator 1 adds a new module to the group of modules that make up the system. The degrees of freedom of the system are increased, and higher functionality can be realized. Operator 2 is an operator that alters the properties of a given module. Concerning operator 2 , modification of link shapes by operator $2-1$ and of the number of joints by operator 2-2 can be used any number of times.

Examples of system modifications by these operator actions are shown in Fig.3. The change from system $f_{1}$ to system $f_{2}$ is an example where operator 1 is applied to add a module. The linked mechanism is changed from having one degree of freedom to two degrees of freedom for the serial drive type mechanism (manipulator). The change from system $f_{2}$ to $f_{3}$ corresponds to the modifications of the internal variables of links. The action of operator 2-1 where a joint is added to a link produces a parallel drive type manipulator having two motors on a pedestal. By using these two kinds of operators, a variety of systems can be expressed using combinations of modules.

The requirements set by the user are: 1) minimization of consumed energy and 2) maximization of the operational simplicity of the link mechanism. The user's requirements concerning the amount of energy consumed and the dynamic manipulability are used as the criteria for this product design.

The consumed energy is calculated from the magnitudes of torques applied to the joints. The requirement concerning the consumed energy is expressed by minimizing the summation $f_{1}$ of the consumed energy of each motor over all motors, while the manipulability requirement is expressed by minimizing 


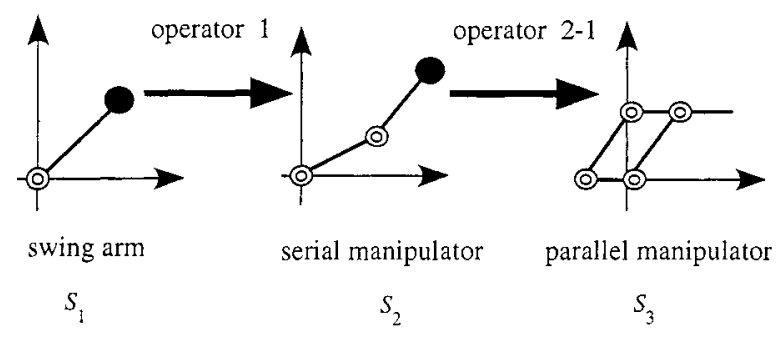

Figure 3. Examples of operator actions

the summation $f_{2}$ of the reciprocal of the dynamic manipulability measure $\omega_{d_{j}}$ over the all measuring points of the system. The objective functions $f_{1}$ and $f_{2}$ concerning the consumed energy and the manipulability are respectively formulated [3].

Fig.4 shows an example of a tree diagram for a design that was constructed and later stored in a database. Fig.5 shows changes in Pareto optimum solutions for the example. The history of the tree formation shown in Fig. 4 is explained as follows. Operator $i(i=1,2-1$, and 2-2) between various nodes represents operators active in the generation of subsequent systems. Operator type 1 alters the combination of modules, while type 2 operators change internal variables. Here, operator 2-1 changes the number of joints of the link, while operator 2-2 changes the position of the center of mass. Both operators 2-1 and 2-2 change internal variables of a given module.

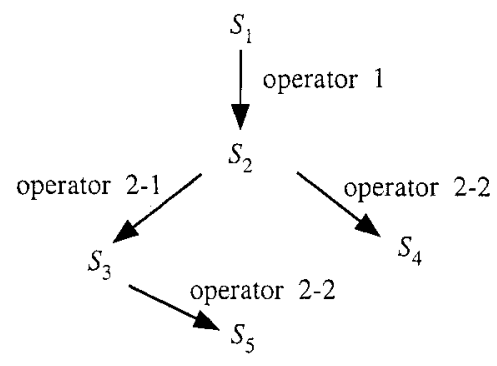

Figure 4. Tree form data of design solutions

First of all, from a unit link mechanism $S_{1}$, a serial drive type manipulator $S_{2}$ was obtained by the addition of a module. Next, the action of operator 2-1 upon $S_{2}$ generated $S_{3}$, a parallel type drive manipulator, while applying operator 2-2 to $S_{2}$ yielded $S_{3}$, in which the center of mass was changed by changing the cross-sectional shapes of the links. After evaluation of $S_{3}$ and $S_{4}, S_{5}$ was 


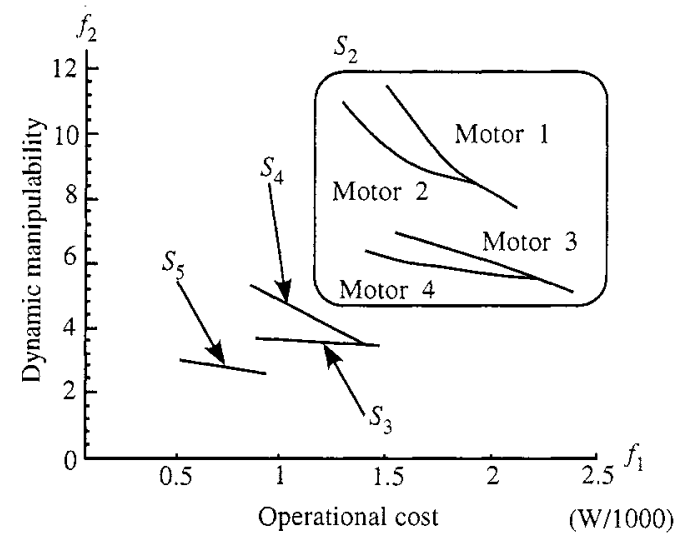

Figure 5. Changes in Pareto optimum solutions

obtained by applying operator 2-2 to $S_{3}$. But $S_{4}$ became a dead-end, since the action of any operator upon $S_{4}$ generated an identical system. For the same reason, $S_{5}$ became a dead-end. Ultimately, the tree-form data shown in Fig.4 was obtained.

Next, the changes in Pareto optimum solutions during the design generation processes are shown in Fig.5. Fig.5 is a space showing two criteria, $f_{1}$ and $f_{2}$, where the Pareto optimum solution set for each generated system is displayed. Since smaller magnitudes are preferable for each of the criteria, the design solution near the origin of the coordinates in the criteria space shown in Fig.5 is more preferable. For $S_{2}$, features of optimized results including discrete design variables of four kinds of motors are displayed for each kind of motor. For each of the other systems, the best Pareto optimum solution is shown. It can be understood from Fig.5 that the order of preferable solution lines is $S_{5}$, $S_{3}, S_{4}$, and $S_{2}$.

At this time, examining the generational history represented by the tree form diagram shown in Fig.4 will aid understanding the solution sequence, since the origin of the obtained preferable solution can be seen. From Fig.5, the optimum solution set for the design problem being regarded is $S_{5}$. The design system is a parallel drive type manipulator having two links.

\subsection{Concurrent evaluations and optimization of related performance characteristics}

Next, product performances that are related to the product design are concurrently optimized at the product design planning stage. For this purpose, a hierarchically decomposed structure of multiobjective optimization problems 
having multiple performance characteristics is displayed, and corresponding relations among hierarchical Pareto optimum solutions are obtained to aid a deeper understanding of optimized solutions [4] [5]. A hierarchical multiobjective optimization method is one in which multiobjective optimization models are hierarchically constructed.

Characteristics expressing product performance are here included in the objective functions when the multiobjective optimization problem is formulated. The characteristics are here called "performance characteristics". When each characteristic in a group of characteristics has individually different optimum design solutions, the characteristics of the group will have conflicting interrelationships during the optimization of the system as a whole. Generally, the group of characteristics included in the objective functions has conflicting interrelationships.

\section{(i) Hierarchical construction of optimization problems}

In the first stage of the proposed product design optimization method, each performance characteristic in the group of product performances is decomposed into simpler basic characteristics according to its structure. Alternatively, simpler characteristics are extracted from performance characteristics, to accommodate the specific features, or difficulties, of the particular design problem. Decomposition and extraction techniques are sequentially applied until the characteristics become sufficiently simple to use in the next stage of the procedure. The decomposed and extracted characteristics are placed in hierarchical levels that are below those of the original characteristics. The decomposed or extracted characteristics are here simply called "characteristics" to distinguish them from performance characteristics.

In this research, the decomposed or extracted characteristics and design variables are ordered in a hierarchical structure, creating a hierarchical display of system components, based on the clarification of input and output relationships among the components comprising the system. This ultimately provides an easily understandable global view of the system as a whole, such as is shown in Fig.6. The construction of optimization strategies is then based on this global structural model.

Characteristics on the same hierarchical level have different input variables. The set of characteristics sharing common input variables is denoted as a basic optimal unit group.

In Fig.6, characteristics $f_{7}$ and $f_{8}$ have common design variables, vector $\mathbf{d}_{1}$ , while characteristics $f_{9}$ and $f_{10}$ have common design variables, vector $\mathrm{d}_{2}$. In such cases, $f_{7}$ and $f_{8}$, and $f_{9}$ and $f_{10}$ are respectively unified as basic optimum unit groups. $f_{3}$ and $f_{4}$ have common input variables, namely $f_{7}, f_{8}, f_{9}$ and $f_{10}$. In such cases, $f_{3}$ and $f_{4}$ are unified as a basic optimum unit group at a higher hierarchical level. Characteristics existing in the same basic optimal unit group are essentially simultaneously optimized as a multiobjective optimization 


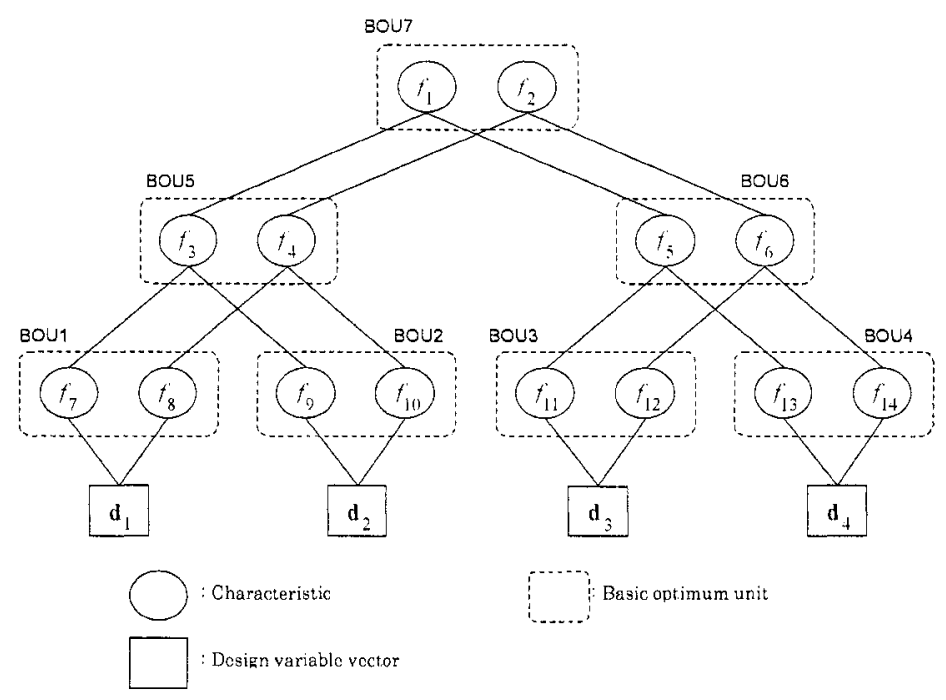

Figure 6. Hierarchical construction of the optimization problem

problem. Optimizations start at the bottom level of the basic optimal unit groups, for example, $f_{7}$ and $f_{8}$, and then proceed to the higher levels. Basic optimum units existing at the same hierarchical level can be optimized separately or concurrently as needed, reducing the required computation time.

The Pareto optimum design solutions obtained in a basic optimal unit group are included in the input variables for the optimization of basic optimal unit groups located at higher levels along the decomposition path. Here, design solutions at discrete points on the Pareto optimum solution set are transferred for use in upper level optimizations. The Pareto optimum solutions obtained by each optimization are added one after another, to obtain Pareto optimum solutions for the whole basic optimal unit group. Finally, the Pareto optimum solutions at the top hierarchical level are achieved.

\section{(ii) Deeper insight into the results of design optimization}

The results derived from the design optimization are only solutions obtained based on the initially given formulations. Even if multidisciplinary optimizations are applied, it is impossible to include all the product design factors in the initial formulations. Optimization methods should not simply be used just to obtain final design solutions to the problem at hand, but also for effectively and rationally obtaining candidate design solutions for further design investigations and improvement. The information and knowledge obtained by the design optimization should, ideally, be used as investigational data for further design improvements. 
One of the advantages of the hierarchical optimization method proposed in this paper is that it allows explicit investigation of Pareto optimum solutions at the lower hierarchical levels, leading to deeper insight into the results of design optimization and improved optimization formulations so that superior design solutions can be obtained.

Designers can assess the corresponding relationships between a design solution selected from the Pareto optimum solution set at the highest hierarchical level of the optimization, and a design point on a Pareto optimum solution set at a lower hierarchical level. Fig.7 shows the correspondence of various design solution points on the Pareto optimum solution set curves at different hierarchical levels. In Fig.6, BOU7 is composed of characteristics $f_{1}$ and $f_{2}$. Point $\mathrm{A}$ on the BOU7 Pareto optimum solution curve corresponds to both point $\mathrm{B}$ on the BOU5 Pareto optimum solution curve and point $\mathrm{C}$ on the BOU6 Pareto optimum solution curve. Furthermore, at the lower hierarchical level, point A corresponds to point $\mathrm{D}$ on the BOU1, point $\mathrm{E}$ on the BOU2, point $\mathrm{F}$ on the BOU3, and point $\mathrm{G}$ on the BOU4 Pareto optimum solution curves. Such detailed clarification of corresponding design points is a useful and important feature of the proposed method.

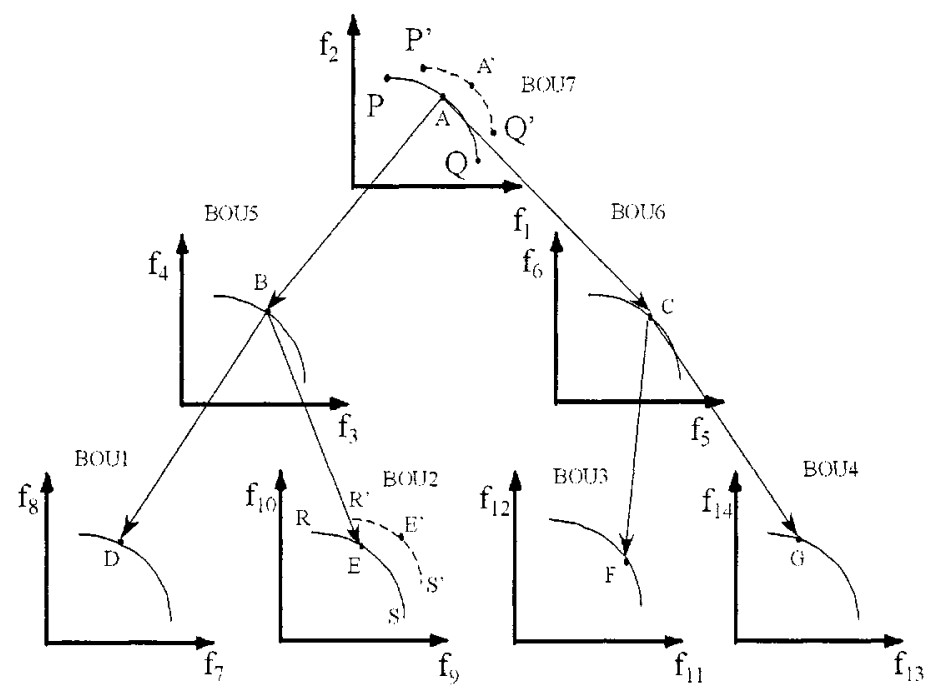

Figure 7. Correspondence of design solution points

In Fig.7, given the conflicting relationship of the essential characteristics $f_{9}$ and $f_{10}$ at the lowest hierarchical level of the optimization problem, the breakthrough design alternative that yields the improved Pareto optimum solution shown by the dashed line R'S' can now be considered. When the new 
$f_{9} / f_{10}$ Pareto optimum solution is applied during further optimization, a new, enhanced Pareto optimum solution line for performance characteristics $f_{1}$ and $f_{2}$ at the highest hierarchical level is obtained, indicated by the dashed line P'Q' [4] [5].

\section{Concluding Remarks}

In current product design scenarios, rapidly changing customer preferences make reductions in product development/design time a practical necessity for many companies. Furthermore, competition among companies for products that can be offered at lower cost, while providing better performance and quality, higher reliability, and so on, is relentless and ongoing. Since product cost and product performances/characteristics are essentially determined at the product planning stage, the success or failure of product development depends on how appropriately optimization methods can be applied at this stage. As explained in this paper, many alternative designs should be generated and quickly compared at the product planning stage, and the relationships among associated characteristics should be effectively evaluated. The application of multiobjective optimization methods where Pareto optimum solution sets are obtained and displayed on the characteristics space can be useful and effective.

The methods proposed in this paper, based on the use of particularly sophisticated multiobjective optimization methods, can be effectively used for comparison of many alternative designs and concurrent evaluations of related performance characteristics.

\section{References}

[1] Edited by Stadler, W., 1988, Multicriteria Optimization in Engineering and in the Sciences, Plenum Press.

[2] Edited by Eschenauer, H, Koski, J, and Osyczka, A., 1990, Multicriteria Design Optimization, Springer-Verlag.

[3] M. Yoshimura and S. Horie: Concurrent Design of Mechanical Systems Using OperatorActing Modules, JSME International Journal, Vol.43, No.2, Series C, 2000, pp.478-485.

[4] M. Yoshimura, K. Izui, S. Komori, and S. Nishiwaki: Breakthrough of Design Solutions Enabled by Extraction of Core Factors in Product Design Optimization, Proceedings of the fifth World Congress of Structural and Multidisciplinary Optimization, Paper No.82, 2003.

[5] M. Yoshimura, M. Taniguchi, K. Izui, and S. Nishiwaki: Hierarchical Arrangement of Characteristics in Product Design Optimization, ASME Journal of Mechanical Design (in process of publication). 